\title{
The Physical Optimum as an Ideal Reference Value for Balancing Thermodynamic Processes Integrating the Exergetic Evaluation by the Example of Heat Supply
}

\author{
Dirk Volta * and Samanta A. Weber \\ Department of Energy and Biotechnology, Flensburg University of Applied Sciences (FUAS), \\ 24943 Flensburg, Germany; samanta.weber@hs-flensburg.de \\ * Correspondence: dirk.volta@hs-flensburg.de
}

Citation: Volta, D.; Weber, S.A. The

Physical Optimum as an Ideal

Reference Value for Balancing

Thermodynamic Processes

Integrating the Exergetic Evaluation by the Example of Heat Supply. Energies 2021, 14, 4426. https:// doi.org/10.3390/en14154426

Academic Editor: Xiaolin Wang

Received: 16 June 2021

Accepted: 19 July 2021

Published: 22 July 2021

Publisher's Note: MDPI stays neutral with regard to jurisdictional claims in published maps and institutional affiliations.

Copyright: (c) 2021 by the authors. Licensee MDPI, Basel, Switzerland. This article is an open access article distributed under the terms and conditions of the Creative Commons Attribution (CC BY) license (https:// creativecommons.org/licenses/by/ $4.0 /)$.

\begin{abstract}
This paper contains the basic definition and application of the physical optimum as a method for process evaluation and optimization. By means of the exemplary balance of a wood pellet-fired boiler, the conventional efficiency is compared to the PhO. Furthermore, this study demonstrates the possibility of applying the thermodynamic state variable exergy as a physical reference property of a system within the $\mathrm{PhO}$ method. To explain the approach, the heat generation in the wood pellet-fired boiler is compared to the supply from a heat pump, which itself is connected to a power plant. Furthermore, the process-independent $\mathrm{PhO}$ is explained in order to illustrate the limitations of feasible optimization. Additionally, possible research topics such as the integration of dynamic behavior in the method are approached. As a conclusion, the differences between the methods outline the advantage of the $\mathrm{PhO}$ in the optimization process.
\end{abstract}

Keywords: physical optimum; $\mathrm{PhO}$; reference process; exergy; energy efficiency; energy optimization; method comparison

\section{Introduction}

Technical analysis often uses comparison models in order to describe a process in a simplified manner, and, additionally, to compare the model to a real process with a certain degree of energy loss. The literature provides different terms for the reference process. The following list illustrating the existing variety shows a range of possibilities for process evaluation [1]:

- BDP: best demonstrated practice;

- OEO: operational energy optimum;

- PEO: plant energy optimum;

- $\quad$ TEO: theoretical energy optimum;

- $\quad$ Best practice observed;

- BAT: best available technology;

The method of the physical optimum $(\mathrm{PhO})$ was primarily described in the dissertation of Volta [1]. Keichel provided an additional consideration in his dissertation [2] in 2017 as well as the resulting VDI 4663 [3], which is mainly based on these studies. Wenzel [4] provided a systematic comparison of the $\mathrm{PhO}$ to the exergy method. Kerpen [5] provided a differentiation between the $\mathrm{PhO}$ and exergetic evaluation.

This study explains the physical optimum and the $\mathrm{PhO}$ factor $F_{P h O}$ as the corresponding indicators and integrates the already common methods of efficiency and exergy analysis, using the methods in combination. The particularity of the application of the $\mathrm{PhO}$ is the reference to an ultimate value. This allows the analysis of losses, aiming to distinguish whether they are avoidable or not because an optimization can only reduce avoidable losses. Other studies such as [4,5] outlined the differences between the $\mathrm{PhO}$ and 
other evaluation methods, while this study aims to integrate, in particular, the state value exergy into the $\mathrm{PhO}$.

\section{Materials and Methods}

Below, the approaches to energy optimization relevant to this study are explained, namely, efficiency, exergy analysis and the $\mathrm{PhO}$. The distinct characteristics of the methods are outlined.

\subsection{Efficiency}

The efficiency is defined as the output-to-input ratio of a certain process:

$$
\eta=\frac{\text { output }}{\text { input }}
$$

The efficiency does not specify the conditions under which a process operates. Without further information, it is not possible to draw conclusions on whether the process operates under ideal conditions or not. An efficiency below one only indicates that a part of the expense is not gained as a benefit from the process. Therefore, the degree to which any losses result, in general, becomes evident.

Thus, the efficiency is a fundamental application of the first law of thermodynamics, namely, energy conservation.

\subsection{Exergy Analysis}

For the state variable exergy $E$, the determination of a ratio in analogy to the efficiency is typical. The exergetic efficiency is defined as the exergetic benefit in relation to the exergetic expense of a specific process.

$$
\zeta=\frac{E_{\text {output }}}{E_{\text {input }}}=1-\frac{E_{\text {loss }}}{E_{\text {input }}}
$$

with $E_{\text {input }}=E_{\text {output }}+E_{\text {loss }}$.

This study distinguishes between avoidable and unavoidable losses.

$$
E_{l o s s}=E_{\text {loss,avoidable }}+E_{\text {loss, unavoidable }}
$$

If an unavoidable energy flow exits the balance with a temperature differing from the ambient state, an unavoidable exergy flow is transferred to the surroundings in the form of exergy loss. A practical example is a heating process, in which the temperature of a product to be heated is increased over the ambient temperature using a material-bound energy carrier. The following approach considers the exergy of fuels and the exergy of heat for an analytic balance.

Kerpen [5] considered a combustion process as well. The focus in Kerpen's [5] work, however, lies on the explicit difference between the exergy and energy contents of the fuel. The aim of this study is to explain the principle of integrating exergy into the $\mathrm{PhO}$ method. Furthermore, the focus of this study lies on the generation of useful heat including the integration of further applications for energy conversion, not exclusively on the combustion process. Therefore, the example is kept deliberately simple. Thus, in this study, the exergy content of a fuel is described as the fuel performance in a good approximation.

$$
E_{B} \approx Q_{B}
$$

The exergy of heat depends on the amount of heat as well as the ambient temperature in relation to the temperature at which the heat passes the system border [6] (p. 156).

$$
E_{q}=\left(1-\frac{T_{a m b}}{T}\right) \times Q=\eta_{C} \times Q
$$


If the heat is not exchanged at a constant temperature level, meaning the heat transfer is sensible, the average thermodynamic temperature $T_{m}$ of the heat transfer is used to calculate the amount of exergy transferred with the heat. For ideal gases, as well as incompressible fluids with an approximately constant specific isobaric heat capacity, the average thermodynamic temperature can be calculated as follows [6] (pp. 119-120).

$$
T_{m 1,2}=\frac{Q_{12}}{S_{q 12}}=\frac{m \times c_{p} \times\left(T_{2}-T_{1}\right)}{m \times c_{p} \times \ln \left(\frac{T_{2}}{T_{1}}\right)}=\frac{T_{2}-T_{1}}{\ln \left(\frac{T_{2}}{T_{1}}\right)}
$$

\subsection{Physical Optimum}

Below, the $\mathrm{PhO}$ is described based on [1,3]. The value of the $\mathrm{PhO}$ is ultimate for a specific process. To determine the $\mathrm{PhO}$, an ideal thermodynamic reference process must be established for which the application of the laws of thermodynamics is essential. The process can be reversible as well as irreversible. The $\mathrm{PhO}$ is applicable to energy types as well as material and information. Figure 1 shows the $\mathrm{PhO}$ in comparison to common reference processes.

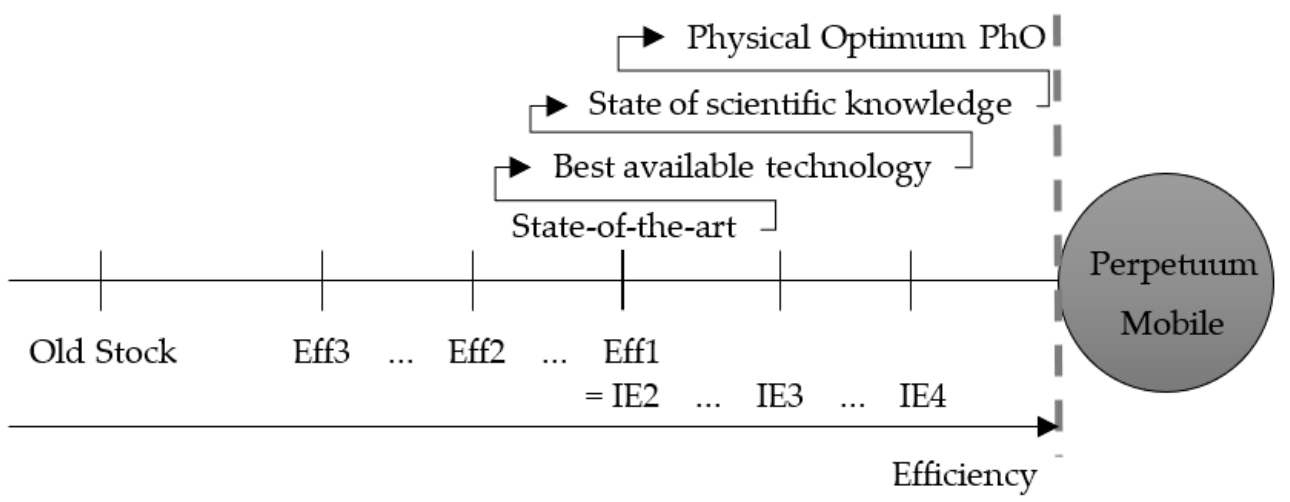

Figure 1. The $\mathrm{PhO}$ in comparison to common reference processes [1].

As an ideal threshold value, the $\mathrm{PhO}$ separates the perpetuum mobile from the processes feasible in reality. The laws of thermodynamics refute the feasibility of a perpetuum mobile. Thus, the perpetuum mobile is also a theoretical concept to define an ideal process, though it is - even as a threshold-not feasible. As an example for the necessity of changing reference points, the efficiency classes of electric motors can be taken into account. Following technical progress, the electric motors according to efficiency class Eff1 were redefined as IE2 to enable the evaluation of further improved motors with IE3 and IE4 [7].

The $\mathrm{PhO}$ factor puts the real amount of energetic expense $B$ in relation to the corresponding physical minimum expense $B_{P h O}$, resulting in a value higher than one at all times (according to the initial approach in [1] to focus on the demand of processes, the symbol " $B$ " is used, as in [3]).

It is therefore necessary to measure the expense $B$, in order to establish it with a simulation, in case the examined process is not running yet. On the other hand, the physical minimum expense $B_{P h O}$ is defined and calculated based on an ideal reference process.

$$
F_{P h O}^{B}=\frac{B}{B_{P h O}} \geq 1
$$

The difference between the $P h O$ factor and the physically optimal case $\left(F_{P h O}^{B}=1\right)$ returns the avoidable losses $\Delta \Omega_{B}$ :

$$
\Delta \Omega_{B}=F_{P h O}^{B}-1
$$


In contrast to the efficiency, the $\mathrm{PhO}$ outlines the avoidable losses, which represents the only possible starting point of process optimization. The efficiency provides the overall loss of a process consisting of avoidable and unavoidable losses. Just for the exceptional case in which the ideal efficiency can reach $1\left(\eta_{\text {ideal }} \rightarrow 1\right)$, the efficiency outlines only the avoidable loss $\Delta \Omega_{B}$ because the unavoidable loss equals zero. In any other case (for $\eta_{\text {ideal }}<1$ ), the efficiency does not enable conclusions regarding the degree to which the process can be optimized because unavoidable losses are included in the overall loss. Below, a practical example of a wood pellet-fired boiler illustrates this problem.

\section{Results}

As an exemplary process, a wood pellet fired-boiler is considered, which provides warm water for space heating with a heat exchanger in the exhaust system. The following calculations are described in a typical way and can be found, e.g., in $[6,8]$.

\subsection{Application of PhO to Boiler Process}

Figure 2 illustrates the boiler system. Fuel $m_{B}$ and combustion air $m_{l}$ are fed to the system. In this model, a complete combustion is assumed.

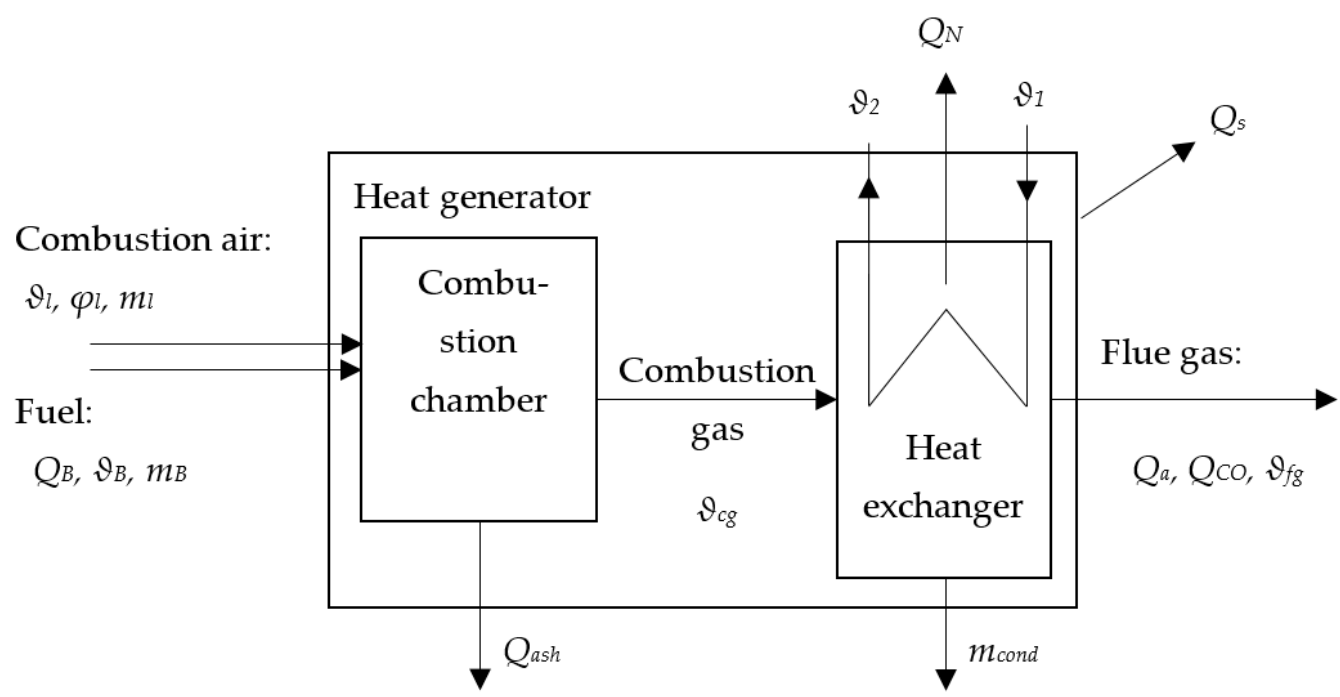

Figure 2. System definition for boiler process.

Resulting from $m_{B}$, the combustion heat $Q_{B}$ is fed to the system. Apart from the useful heat $Q_{N}$, the following losses leave the system:

- $Q_{f g}$ exhaust loss;

- $Q_{a s h}$ ash loss;

- QCO losses by incompletely burned material;

- $Q_{S}$ surface loss.

$$
Q_{B}=Q_{N}+Q_{f g}+Q_{a s h}+Q_{C O}+Q_{s}
$$

A simplified approach to determine the system efficiency is-neglecting the surface loss $Q_{s}$ - the combustion efficiency $\eta_{f}$

$$
\eta_{f}=1-q_{f g}-q_{C O}-q_{a s h}
$$

with

$$
q_{f g}=\frac{Q_{f g}}{Q_{B}} ; q_{C O}=\frac{Q_{C O}}{Q_{B}} ; \text { etc. }
$$


The ash losses as well as the losses by unburned material can typically be neglected in an energetic balance $\left(q_{C O} \rightarrow 0, q_{a s h} \rightarrow 0\right)$. The combustion efficiency is simplified to

$$
\Longrightarrow \eta_{f}=1-q_{f g}
$$

Under the condition of a complete combustion, the exhaust loss is a function of the air ratio $\lambda$, the oxygen concentration in the flue gas and the exhaust temperature:

$$
q_{f g}=f\left(O_{2}^{f g}, \vartheta_{f g}\right)
$$

Furthermore, for complete combustion, the air ratio for fuels with high gross calorific values can be determined in a good approximation according to [8]

$$
\lambda=\frac{C O_{2 m a x}^{f g}}{C O_{2}^{f g}}=\frac{O_{2 m a x}^{f g}}{O_{2 m a x}^{f g}-O_{2}^{f g}}=\frac{0.2093}{0.2093-O_{2}^{f g}}
$$

The specific exhaust loss $q_{g f}$ is defined as

$$
q_{f g, \mathrm{cond}, \mathrm{Hi}}=\frac{\left[\sum\left(v_{i} \cdot C_{m p, i}\right)\right] \times\left(\vartheta_{f g}-\vartheta_{a m b}\right)-w_{\text {cond }} \times r}{H_{i}}
$$

with

$$
\sum_{i=1}^{n}\left(v_{i} \times c\right)=v_{\mathrm{CO}_{2}} \times c_{m p, \mathrm{CO}_{2}}+v_{N_{2}} \times c_{m p, \mathrm{~N}_{2}}+v_{\mathrm{H}_{2} \mathrm{O}} \times c_{m p, \mathrm{H}_{2} \mathrm{O}}+v_{\mathrm{O}_{2}} \times c_{m p, \mathrm{O}_{2}}
$$

It is also possible to refer to the gross calorific value for the combustion efficiency.

$$
\eta_{f, \mathrm{cond}, \mathrm{Hs}}=\eta_{f, \mathrm{cond}, \mathrm{Hi}} \times \frac{H_{i}}{H_{s}}
$$

The specific molar heat capacity of the components in the flue gas $c_{m p, i}$ is approximated for $100{ }^{\circ} \mathrm{C}$ and the condensation enthalpy of water for $25^{\circ} \mathrm{C}$ of $r_{H 2 O}, 2{ }^{\circ} \mathrm{C}=2411 \mathrm{~kJ} / \mathrm{kg}$.

Moreover, the specific flow of condensed water is defined as the flow of the condensate in relation to the supplied flow of the fuel.

$$
w_{\text {cond }}=\frac{\dot{m}_{\text {cond }}}{\dot{m}_{B}}
$$

\subsubsection{Example}

The efficiency of the heat generator depends on $\lambda$ and the exhaust temperature. To determine $\lambda$, a measurement of the following parameters in the flue gas is required (this example is used parallely in a similar form in [3]): $O_{2, f g}=7 \% ; \vartheta_{f g}=130{ }^{\circ} \mathrm{C}$. The real $\lambda$ results in being

$$
\lambda=\frac{O_{2 \max }^{f g}}{O_{2 \max }^{f g}-O_{2}^{f g}}=\frac{0.2093}{0.2093-0.07}=1.5
$$

However, in the case of physically optimal conditions, $\lambda_{P h O}$ would be 1 (complete combustion):

$$
\lambda_{P h O}=\frac{O_{2 \max }^{f g}}{O_{2 \max }^{f g}-O_{2}^{f g}}=\frac{0.2093}{0.2093-0}=1
$$

In addition, the exhaust temperature would equal the lowest temperature in the system (in this case, the return flow temperature at $60^{\circ} \mathrm{C}$ ), resulting in a higher efficiency. 
The following applies: $\vartheta_{1}=\vartheta_{f g}=60{ }^{\circ} \mathrm{C}$, terminal temperature difference TTD $=0$. Figure 3 shows the temperature of the two fluids over the surface of the heat exchanger in the real case and the PhO.

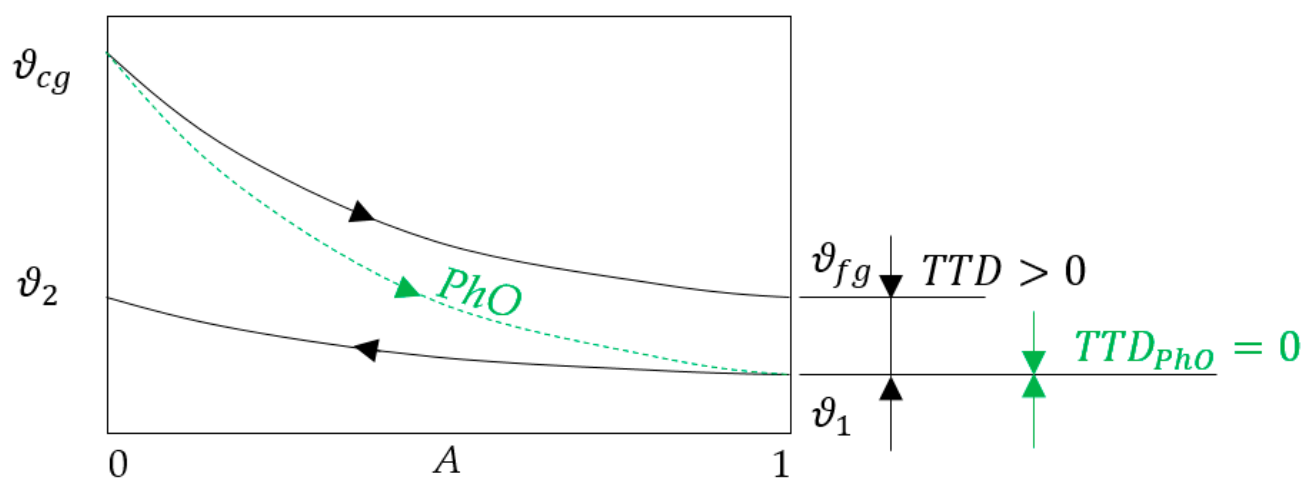

Figure 3. Temperature profile inside the heat exchanger of the heat generator.

A combustion efficiency of $100 \%$ would be impossible to achieve even if the flue gas were cooled back to ambient temperature (in this case, $20^{\circ} \mathrm{C}$ ). The combustion efficiency for a specific temperature with regard to $\lambda$ is shown in Figure 4. (The relative humidity $\varphi$ of the flue gas is about $100 \%$, unlike the relative humidity of the combustion air $(60 \%)$. This is why the combustion efficiency does not reach $100 \%$ in the diagram, even at $20{ }^{\circ} \mathrm{C}$.)

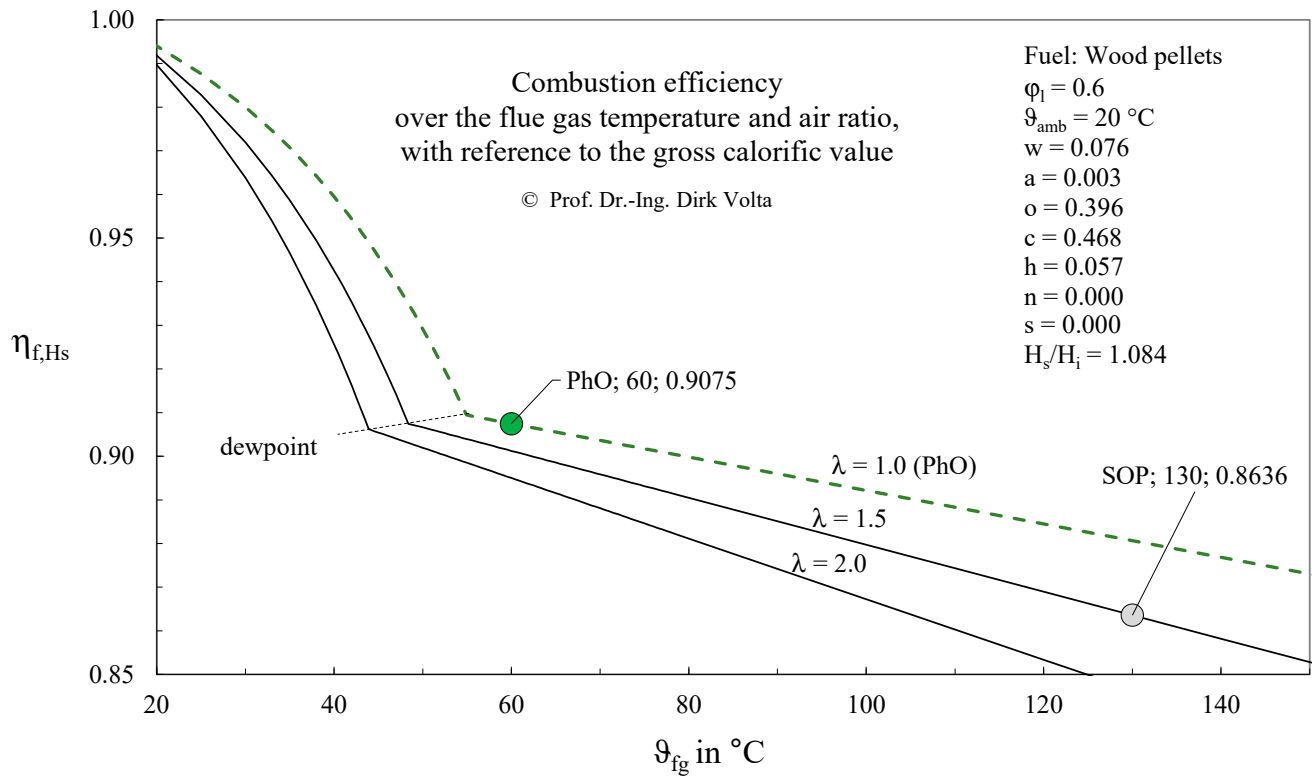

Figure 4. Combustion efficiency for wood pellets, with reference to the gross calorific value.

In the $\mathrm{PhO}$, the combustion efficiency amounts to $90.75 \%$. This corresponds to an exhaust loss which cannot fall below $q_{a, H s, P h O}=9.25 \%$ for this case. Thus, in the $P h O$, if 100 units of fuel are fed to the system, $Q_{N, P h O}=90.75$ units of useful heat can be provided. For the real case, the standard operating point $(S O P)$ is shown in Figure 4. The exhaust loss sums up to $q_{a, H s}=13.64 \%$, which means that it is $q_{a, H s, \Delta \Omega}=4.39 \%$ higher than the ideal case. This equals the potential for optimization. Figures 5 and 6 illustrate the losses. If the input is assumed to be constant, a higher amount of useful heat can be gained for the $\mathrm{PhO}$. Furthermore, the distinction between unavoidable and avoidable losses is shown. 


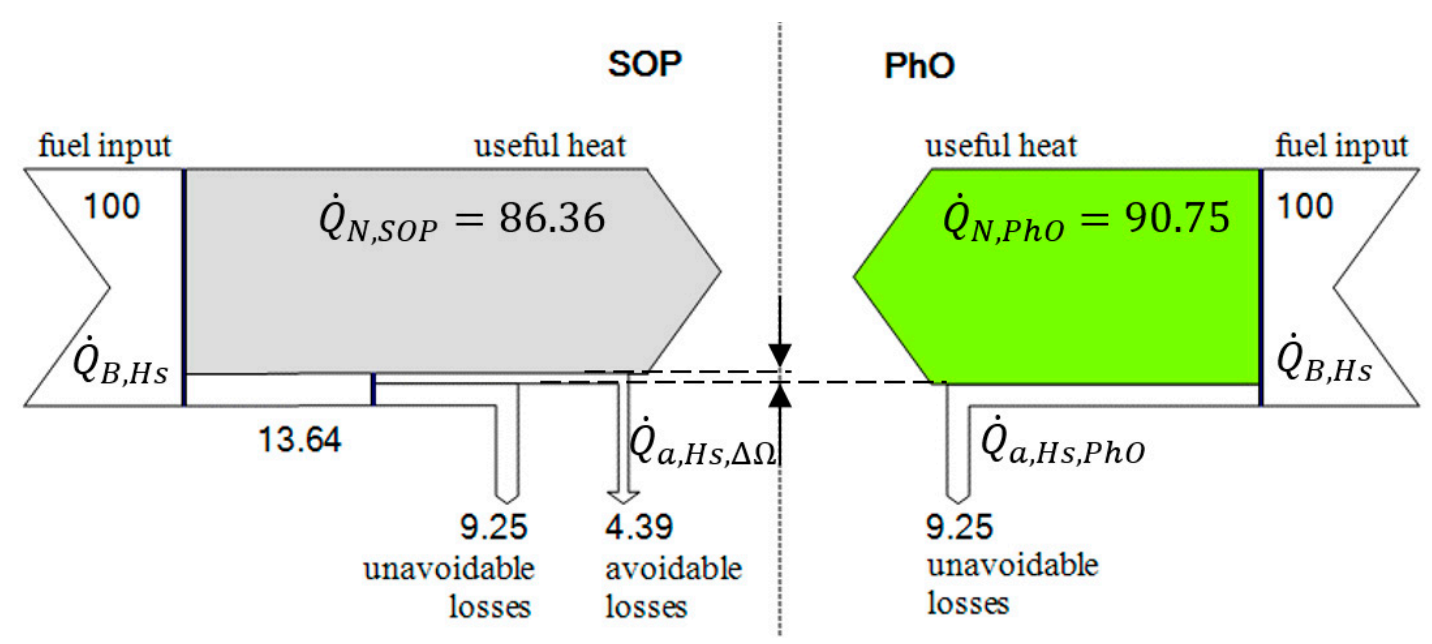

Figure 5. Energy balance.

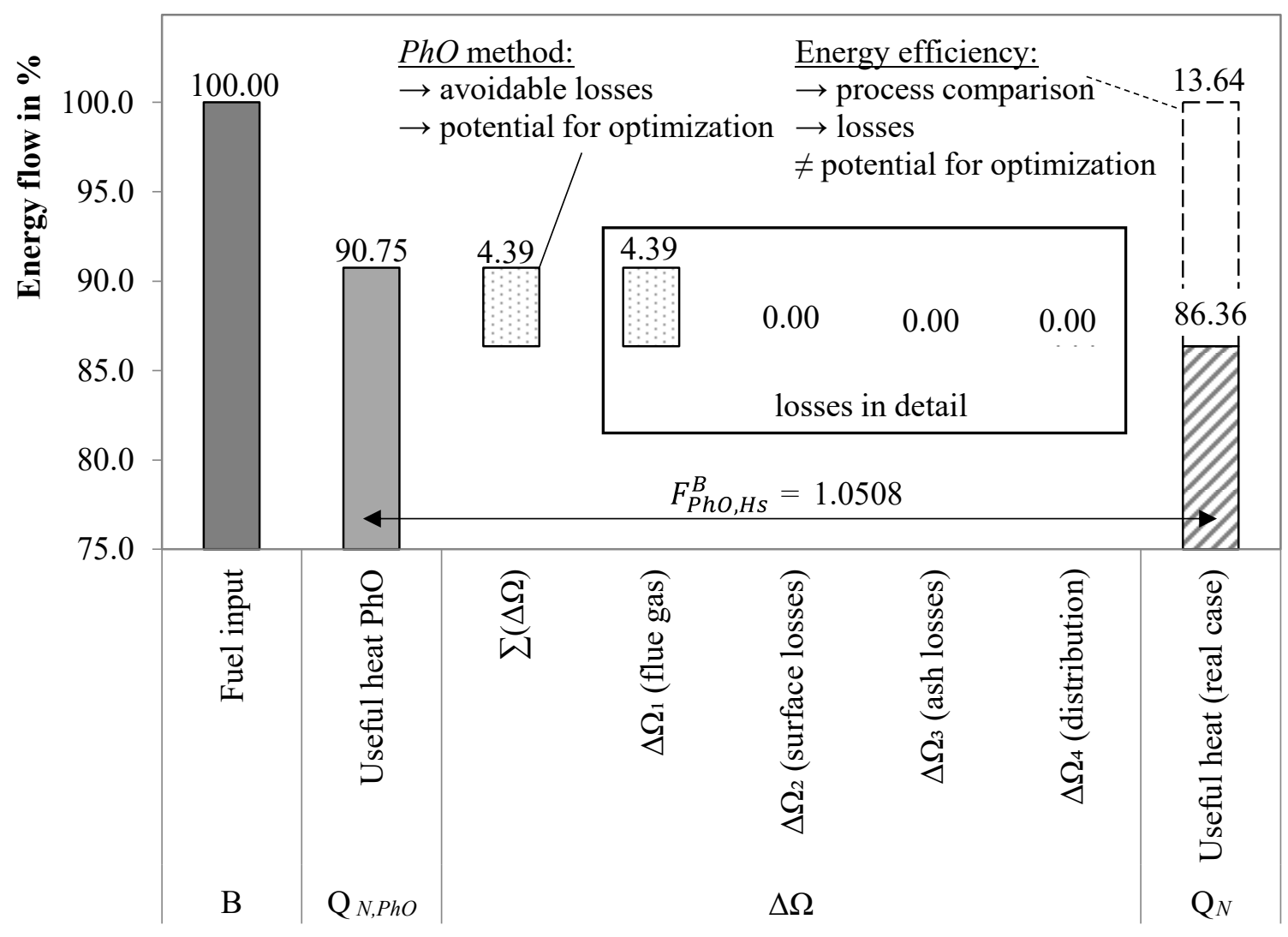

Figure 6. Cascade of losses for boiler.

A more detailed analysis considering further losses would be possible, as indicated in Figure 6.

For the gross calorific value, the following applies:

$$
F_{P h O, H s}^{B}=\frac{B}{B_{P h O}}=\frac{\eta_{f, H s, P h O}}{\eta_{f, H s, S O P}}=1.0508
$$


The avoidable losses equal the potential for optimization. For a specific demand, $105.08 \%$ of the optimal demand is used. In accordance with this, the energy input of the boiler could be reduced by

$$
\Delta \Omega_{B}=F_{P h O, H s}^{B}-1=1.0508-1=5.08 \% \text { - points }
$$

Thus, the avoidable losses do not equal the overall losses:

$$
1-\eta_{f, H s, S O P}=1-0.8636 \rightarrow \text { Potential for optimization } \neq 13.64 \%
$$

It is also possible to determine the $\mathrm{PhO}$ factor for the lower calorific value. For the sampled wood pellets, the ratio of gross and lower calorific values was determined as $H_{s} / H_{i}=1.084$. The combustion efficiency amounts to

$$
\begin{aligned}
F_{\mathrm{PhO}, \mathrm{Hi}}^{B} & =\frac{\eta_{f, \mathrm{Hi}, \mathrm{PhO}}}{\eta_{f, \mathrm{Hi}, \mathrm{SOP}}}=\frac{\eta_{f, H s, \mathrm{PhO}} \times \frac{H_{s}}{H_{i}}}{\eta_{f, H s, \mathrm{SOP}} \times \frac{H_{s}}{H_{i}}}=\frac{\eta_{f, H s, \mathrm{PhO}}}{\eta_{f, H s, \mathrm{SOP}}}=1.0508 \\
& \Longrightarrow \Delta \Omega_{B, H i}=F_{\mathrm{PhO}, \mathrm{Hi}}^{B}-1=5.08 \%-\text { points }
\end{aligned}
$$

Again, the overall losses are higher than the avoidable losses: $1-\eta_{f, H i, S O P}=1-$ $\eta_{f, H i, S O P} \times \frac{H s}{H i}=1-0.8636 \times 1.084=1-0.9359 \rightarrow$ Potential for optimization $\neq 6.41 \%$

However, for both approaches, the potential for optimization amounts to $5.08 \%$-points. Using the $\mathrm{PhO}$ factor, a distinction between the gross and lower calorific values for the evaluation of a combustion process becomes unnecessary. The evaluation of the efficiency with the $\mathrm{PhO}$ factor is valid in general.

\subsubsection{PhO Factor Based on Exergy Analysis}

The ambient temperature is estimated to be $T_{a m b}=293 \mathrm{~K}$. In this study, the outlet temperature of hot water was set to $\vartheta_{2}=80^{\circ} \mathrm{C}$, and the return flow temperature was set to $\vartheta_{1}=60^{\circ} \mathrm{C}$. Thus, the resulting thermodynamic middle temperature is

$$
T_{m}=\frac{353 \mathrm{~K}-333 \mathrm{~K}}{\ln \left(\frac{353 \mathrm{~K}}{333 \mathrm{~K}}\right)}=343 \mathrm{~K}=69.9^{\circ} \mathrm{C}
$$

Real Process:

Explicitly, the exergy of the useful heat in the given example amounts to

$$
\dot{E}_{x, N}=\left(1-\frac{T_{a m b}}{T_{m}}\right) \times \dot{Q}_{N}=\left(1-\frac{293 \mathrm{~K}}{343 \mathrm{~K}}\right) \times 86.36 \text { units }=12.57 \text { units }
$$

Physical Optimum:

The amount of exergy of the flow of useful heat is

$$
\dot{E}_{x, N, P h O}=\left(1-\frac{T_{a m b}}{T}\right) \times \dot{Q}_{N, P h O}=\left(1-\frac{293 \mathrm{~K}}{343 \mathrm{~K}}\right) \times 90.75 \text { units }=13.21 \text { units }
$$

In the real process, the exergetic efficiency is $12.57 \%$. In the case of the $\mathrm{PhO}$, a higher flow of useful heat can be gained at the same temperature level, resulting in a higher exergetic efficiency of $13.21 \%$. Assuming the process conditions are in accordance with the energetic analysis, the same $\mathrm{PhO}$ factor, namely, the same potential for optimization results for the exergetic analysis, is shown in Figure 7. 


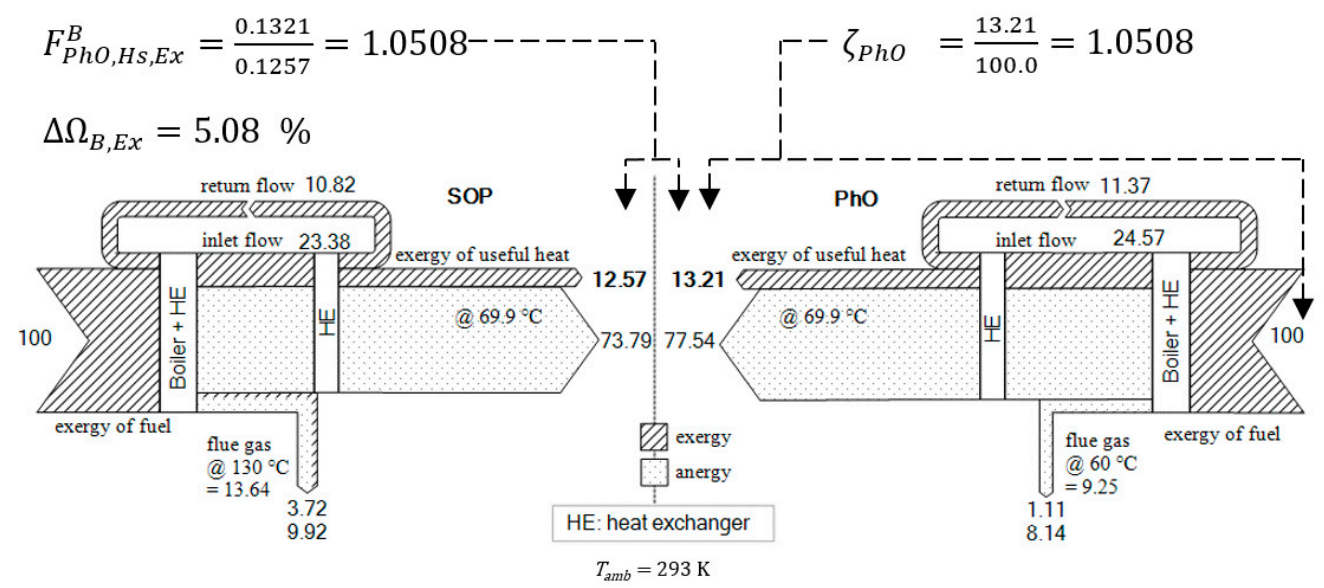

Figure 7. Exergy balance for boiler, real process vs. $\mathrm{PhO}$.

The PhO-Factor based on exergy analysis results in

$$
F_{P h O, H s, E x}^{B}=\frac{B_{E x}}{B_{E x, P h O}}=\frac{\zeta_{P h O}}{\zeta}=\frac{0.1321}{0.1257}=1.0508
$$

The avoidable losses sum up to

$$
\Delta \Omega_{B, E x}=F_{P h O, H s, E x}^{B}-1=1.0508-1=5.08 \%-\text { points }
$$

If the temperature levels are not changed during the optimization (as in this example), the amount of avoidable losses does not depend on the method used to determine this value. The exergetic and energetic balances return the same result. Thus, the determination of the avoidable losses is valid in general: $\Delta \Omega_{B, H i}=\Delta \Omega_{B, H s}=\Delta \Omega_{B, E x}=\Delta \Omega_{B}$.

\subsection{Comparison of Boiler and Heat Pump with PhO}

Comparing the resulting two $\mathrm{PhO}$ factors of the two different processes, the option that is closer to its own ideal consumption is outlined. In this case, the $\mathrm{PhO}$ factor indicates the potential for the optimization of each individual process. It is also possible to use the $\mathrm{PhO}$ to compare different processes with the same benefit, as long as a common ideal comparison process (according to the $\mathrm{PhO}$ ) can be specified for both processes.

In this study, a heat pump process is compared to the wood pellet-fired boiler. The heat pump (HP) is connected to a power plant (PP). Both overall processes are supplied with fuel. Both deliver the same amount of useful energy at the same temperature level.

\subsubsection{PhO Factor and Efficiency}

On the left, Figure 8 shows the boiler process again. Meanwhile, on the right, a simple model of an HP is illustrated. The $\mathrm{PhO}$ for this process can be determined according to Carnot.

- $\quad$ For $B_{P h O}$ : Assuming a TTD of $0 \mathrm{~K}$, the ideal HP operates between 20 and $80{ }^{\circ} \mathrm{C}$, with a COP of 5.88 .

- For $\Delta \Omega_{1}$ : For the real $\mathrm{HP}$, the liquefaction temperature is set to $85^{\circ} \mathrm{C}$, and the evaporation temperature is set to $15^{\circ} \mathrm{C}(T T D=5 \mathrm{~K})$. With a Carnot grade of approximately 0.6 , the COP of the real HP would be 3.07 .

- For $\Delta \Omega_{2}$ : Furthermore, the efficiency of the power plant (PP) delivering the electric energy is assumed to be $40 \%$.

In Figure 8, the $\mathrm{PhO}_{\text {ind }}$ is shown in the middle representing the absolute minimum exergy input for the boiler as well as the HP. The anergy of the useful heat is added in white. In the case of the HP, this anergy is taken from the environment. For the boiler, it is exergy turned into anergy in the process. On the left and right in gray, the consumption in 
the real case is given for each option. In between, the losses are given as a cascade adding to the $P h O_{\text {ind }}$ until the real expense is reached. Thus, the impact of a specific loss on the total losses can be quantified with this form of presentation.

For this process, the $\mathrm{PhO}$ factor sums up to 4.793. As a significant amount of losses are caused by the conversion of fuel to electric energy, the supply with renewable sources should be considered instead. With the losses of the PP practically reduced to zero, the further advantages of the $\mathrm{HP}$ for this application are evident. The $\mathrm{PhO}_{\text {ind }}$ is explained below.

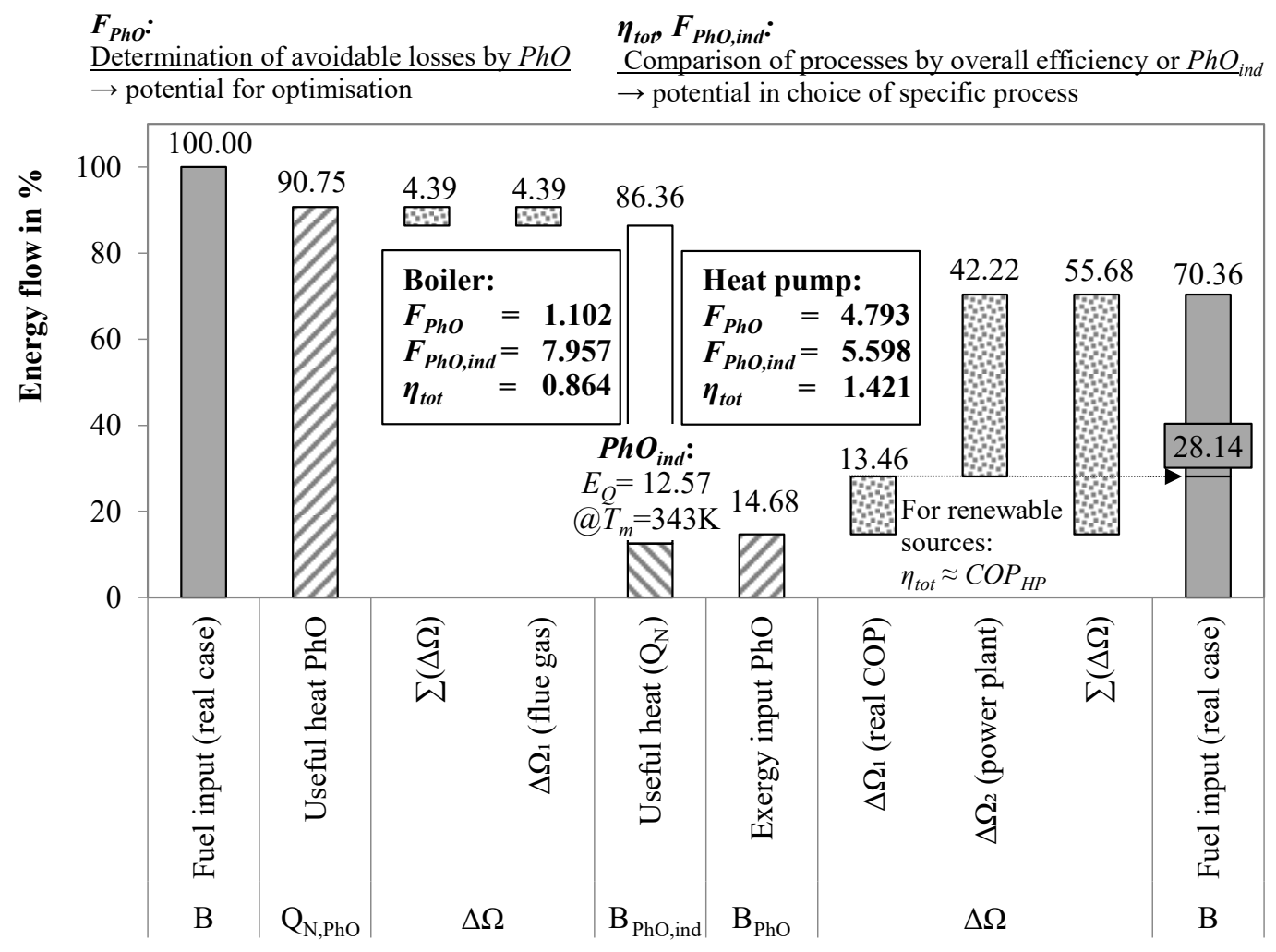

Figure 8. Cascade of losses—comparison of heat supply for boiler and HP.

The overall energy efficiency of the heating considered in this study shall be defined as the ratio of useful heat to input exergy.

$$
\eta_{t o t}=\frac{Q_{N}}{E_{i n}} ; \eta_{t o t, b o i l e r}=\eta_{f} ; \eta_{t o t, H P}=C O P_{H P} \times \eta_{P P}
$$

If the $\mathrm{PhO}$ factors alone are taken into account, the boiler shows little potential for further optimization. However, while the $\mathrm{PhO}$ factor of 1.102 indicates that only $10.2 \%$ of the losses are avoidable, the overall energy efficiency shows that just $89.8 \%$ of the initial energy input can be gained as useful heat from the process. Therefore, the overall fuel demand for the HP is lower, which can be determined by the overall efficiency of 1.421, meaning that the amount of useful heat generated is higher than the fuel input, due to the additional input of pure anergy from the environment. Figure 9 further enhances this. 


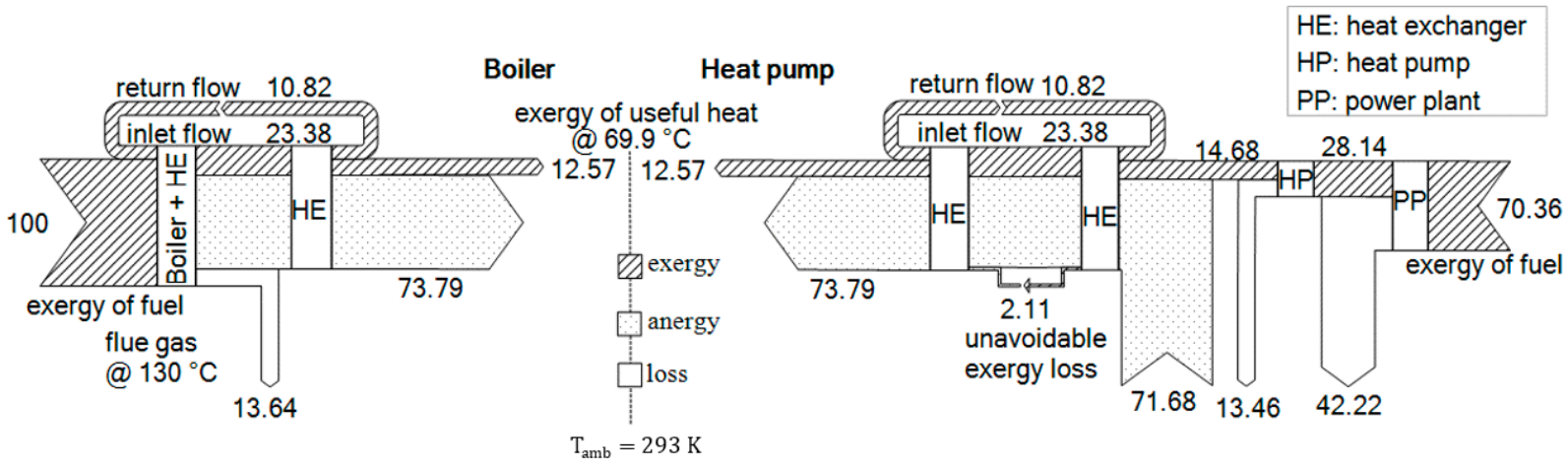

Figure 9. Sankey-comparison of heat supply for boiler and HP.

\subsubsection{Process-Independent $\mathrm{PhO}$}

The $\mathrm{PhO}$ can also be used to compare different processes when referring to an ultimate, process-independent $\mathrm{PhO}\left(\mathrm{PhO}_{\text {ind }}\right)$, which is also shown in Figure 8 . The minimum amount of exergy needed to increase the temperature from 60 to $80^{\circ} \mathrm{C}$ can be determined by the exergy in the transferred heat of 12.57 units. This equals the amount of exergy transferred to the flow of heating water in both options. In relation to the $\mathrm{PhO}_{\text {ind }}$, it is possible to determine a process-independent $\mathrm{PhO}$ factor $F_{P h O, i n d}$. In this case, the HP is the more efficient option while simultaneously consuming less energy, as shown by the factors.

$$
\begin{gathered}
F_{P h O, \text { ind,Ex,boiler }}=\frac{B_{\text {boiler }, E x}}{B_{P h O, \text { ind, }, E x}}=\frac{100}{12.57}=7.96 \\
F_{P h O, \text { ind,Ex,HP }}=\frac{B_{H P, E x}}{B_{P h O, \text { ind,Ex }}}=\frac{70.36}{12.57}=5.60
\end{gathered}
$$

However, the $\mathrm{PhO}$ for a specific process exclusively provides valid information on the degree of possible optimization. Figure 10 compares the temperature levels (of the two fluids) of the real heat transfer (I.), the $\mathrm{PhO}$ (II.) and the process $\mathrm{PhO}_{\text {ind }}$ (III.).

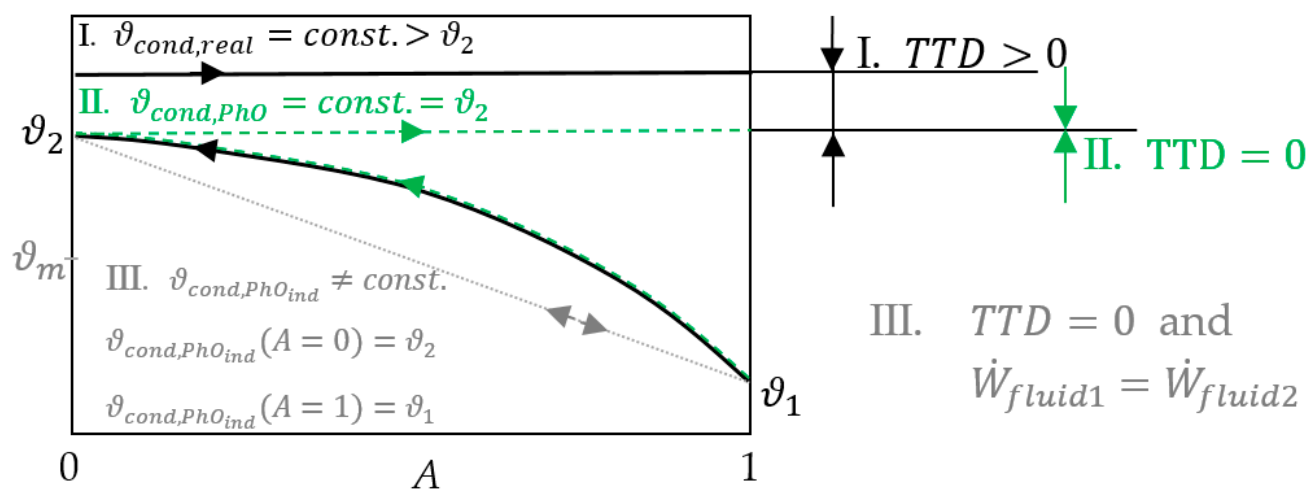

Figure 10. Temperature profile of the heat transfer in the condenser of the $\mathrm{HP}$ (real vs. $\mathrm{PhO}, \mathrm{PhO}_{\text {ind }}$ ).

The process-independent ideal heat transfer is reversible, meaning the temperature difference between the two fluids in the heat exchanger is zero at all times. Even in theory, this is only possible if the thermal capacity flow $\dot{W}=\dot{m} \times c_{p}$ of the heat supply (fluid 1) equals that of the heat demand (fluid 2), as shown in Figure 10. The temperature $\vartheta_{\text {cond,PhOind }}$ therefore has to change during condensation to reach $\vartheta_{2}$. As this is not feasible for a phasechanging single-component fluid at constant pressure in reality, the difference between the $\mathrm{PhO}$ of this process and the $\mathrm{PhO}_{\text {ind }}$ is an unavoidable loss. A heat pump operating between ambient temperature and the thermodynamic middle temperature $\vartheta_{m}$ returns the result for the exergetic expense. Yet, $\vartheta_{m}$ is below the required temperature $\vartheta_{2}$. A condensation 
at $\vartheta_{m}=$ const. cannot supply the system. It becomes clear that in this case, the $P h \mathrm{O}_{\text {ind }}$ is over-idealized, outlining the reversible heat transfer, which is not feasible in reality.

To sum up, the $\mathrm{PhO}$ ind is suitable for a process comparison and, as with the efficiency, determines the process with a lower total energy consumption. The $\mathrm{PhO}$ factor, in addition, outlines the efficiency of each specified process as well as the potential for optimization.

\section{Discussion}

Many indicators for the evaluation of processes exist. To evaluate the efficiency of a process, the $\mathrm{PhO}$ is one of those indicators. The following section discusses the advantages and particularities of the $\mathrm{PhO}$ in order to put the method into the context of research on optimization.

\subsection{Degree of Idealization}

To determine the $\mathrm{PhO}$, a certain process is idealized. Some improvements can only be reached with infinite expense through optimization, such as the TTD of $0 \mathrm{~K}$. The surface of the heat exchanger would have to be infinite. In some cases, it can be reasonable to use a realistic TTD instead. If specific parameters are definite, the $\mathrm{PhO}$ can be determined taking them into account. An example would be a safety margin or a minimum temperature requested (keyword legionella). The system boundaries as well as the idealization of the process must be executed considering the purpose of determining the feasible minimum input. When over-idealized, as in Figure 10, the outcome of the analysis is not valid anymore. While the perfect heat exchanger would run as a reversible process, the user of the $\mathrm{PhO}$ method should consider finding a more realistic model.

It would have been possible to compare the real HP to a model according to Carnot, which takes into account the TTD of $5 \mathrm{~K}$ for each heat exchanger. Then, of course, the minimum temperature for the flue gas of the boiler should also be determined with a realistic TTD. Yet, the TTD should not be changed once specified. The PhO must be an ultimate value. Therefore, the determination of the boundary conditions is decisive. Overall, the definition of the $\mathrm{PhO}$ depends on the purpose of the analysis. The heat exchanger can thus be defined in different ways, as shown in the example in Figure 10. A reason for the chosen description should be given at all times. The distinction between avoidable and unavoidable losses shows the effect of the $\mathrm{PhO}$ being defined closer to the actual process conditions: losses which are avoidable for the idealized version are unavoidable for the more realistic one. Losses occur in both cases. Thus, the consideration must always be whether this categorization has been performed according to the aim of the analysis.

\subsection{Description of the Benefit of a Process}

It is important to define the benefit of a process. Especially for the efficiency, the definition becomes difficult if a process does not generate useful energy, but rather, e.g., a material product. For the heating purpose in this study, the minimum input is easily outlined by the demand as the specific or absolute amount of exergy needed to raise the temperature of the heating water. However, for example, in a baking process, heat is also required. Still, the benefit is the baking of the product, not necessarily the exergy it contains leaving the oven. If the exergy in the product is evaluated as a loss, the energetic and exergetic efficiency would be zero, although the benefit is achieved. The $\mathrm{PhO}$, on the other hand, can be defined, in this case, as the minimum requested energy, for example, to reach a specific temperature. Thus, the $P h O$ factor would return a distinctive value, unlike the efficiency, enabling the evaluation of the efficiency. The $\mathrm{PhO}$ allows analysis of a wide range of different applications and is not bound to specific processes, as with some other existing indicators. 


\subsection{Using Exergy to Describe the PhO}

Taking the exergy into account as a state variable enables a more detailed description of a process in some cases. As it was shown for the boiler, the $\mathrm{PhO}$ evaluation by means of the exergy confirms the results of the energetic evaluation. Anyway, comparing the $\mathrm{HP}$ to the boiler, the exergy analysis shows the absolute minimum input in both cases, which allows a direct comparison. If the energy stored in the fuel is directly compared to the electric energy fed to the HP, the quality of the energy is not regarded. The exergetic content of a specific form of energy can be of high significance for a process comparison. Comparing heat to electric energy means comparing a combination of exergy and anergy to pure exergy. For some applications, the consideration of the exergetic content is highly essential. Thus, the exergy analysis should be integrated into the $\mathrm{PhO}$ when necessary.

\subsection{Research Directions: Change in Load}

The given examples for the $\mathrm{PhO}$ describe stationary processes. A change in load was not taken into account in this study. Though it is possible to consider this state as well, this only makes sense if the process is indeed dynamic. If the essential period of time can be outlined, the description of the dynamics in an ideal model is possible again, meaning a $\mathrm{PhO}$ can be defined for the change in load as well. The losses caused by the change in load show up in the efficiency of a process over a longer period as well (e.g., seasonal COP). The loss can be significant in a certain instant, and yet be small in the long term due to the short period of time in which it occurs. For a dynamic process such as transport, an optimization of the dynamic behavior can be reasonable. If the dynamics are not taken into account for the $\mathrm{PhO}$, they would show up as avoidable losses in the $\mathrm{PhO}$ factor. Therefore, again, the application determines the required level of detail of the analysis.

\subsection{Application of the PhO to Other Examples}

For a process, the description of the $\mathrm{PhO}$ depends on the aim of the analyses, as it has been shown. There are several possibilities for ideal reference processes. The $\mathrm{PhO}_{\text {ind }}$ in comparison to the $\mathrm{PhO}$ of each process is an example for this circumstance. However, this study is limited to the example of two options for the supply of heat. In general, the principle can be transferred to other processes. The VDI 4663-1 [3] provides further examples for the application of the $P h O$ as well as the references $[1,2,4,5]$. As for any other method, the application of the $\mathrm{PhO}$ is limited. An example is a process which does not need any input in the ideal case: Considering a room with a temperature differing from the ambient state, an expense of energy would be needed to balance the heat transfer between the environment and the room and maintain the temperature. For an ideal case, the room would be ideally isolated, resulting in an adiabatic system border. The temperature in the room would not change. Thus, the ideal input is zero. The $P h O$ factor is not applicable, $F_{P h O} \rightarrow \infty$. This particular case could be a potential for improvement in the PhO method.

\section{Conclusions}

This study presented the potentials of the $P h O$ as well as some particularities and research opportunities. In particular, the advantages of the $\mathrm{PhO}$ as a method for energetic analysis were outlined.

Firstly, the $\mathrm{PhO}$ establishes the principle of a distinction between avoidable and unavoidable losses. The efficiency only indicates the occurrence of losses in general. The comparison between the efficiencies for the two calorific values outlines one of the advantages of the $\mathrm{PhO}$. While the efficiency depends on the reference to the gross or lower heating value, the $\mathrm{PhO}$ method indicates the same degree of avoidable losses in both cases.

Secondly, the integration of exergy as a state variable enables a more precise description of the process for the determination of the $\mathrm{PhO}$. The limits of energy conversion are taken into account by evaluating a type of energy by its exergy content. For the boiler, most of the exergy of the fuel was turned into anergy. Meanwhile, pure anergy was turned into 
useful heat by a certain amount of exergy in the HP. The distinction between exergy and anergy allows, in this case, a more detailed comparison of the HP to the boiler.

Thirdly, the $\mathrm{PhO}_{\text {ind }}$ enables a direct comparison of different processes in order to outline not only the potential for optimization but also an overall energy input. For the $\mathrm{PhO}$ factor, a real demand is considered in relation to an ideal input, which means that, for example, the type of energy is the same for both. The COP of an HP compares heat to pure exergy, and the combustion efficiency compares useful heat to the exergy of the fuel. On the other hand, for the $\mathrm{PhO}$ factor, the exergy content of the real input energy type equals that of the ideal input. Thus, the $\mathrm{PhO}_{\text {ind }}$ factors can be compared directly. This also outlines the limits of optimization. For an over-idealized reference, unavoidable losses are categorized as avoidable ones.

Additionally, the $\mathrm{PhO}$ allows the consideration of upstream processes, such as the PP providing the electric energy for the HP. The HP process itself might not enable further optimization, while the upstream processes do. In this case, the option of integrating renewables was illustrated for the HP. The example in this study was kept deliberately simple to demonstrate the principle which can be applied to more complex systems.

Lastly, this study pointed out the perspective of further research projects of the $\mathrm{PhO}$ such as a change in load.

The significance of reducing the environmental impact of processes is not only evident in inhibiting climate change but also under all conditions. For process optimization, it is inevitable to determine the avoidable losses. The method of $\mathrm{PhO}$ is particularly suitable for this. The $\mathrm{PhO}$ defines a practical and user-oriented method for the application to a wide range of processes. Therefore, the $\mathrm{PhO}$ contributes to the progress in optimization in particular, and the primary objective of resource efficiency in general.

Author Contributions: Conceptualization, D.V. and S.A.W.; methodology, D.V. and S.A.W.; software, D.V. and S.A.W.; validation, D.V. and S.A.W.; formal analysis, D.V. and S.A.W.; investigation, D.V. and S.A.W.; resources, D.V.; data curation, D.V.; writing—original draft preparation, D.V. and S.A.W.; writing-review and editing, D.V. and S.A.W.; visualization, D.V. an S.A.W.; supervision, D.V.; project administration, D.V.; funding acquisition, D.V. All authors have read and agreed to the published version of the manuscript.

Funding: This research was funded by the Evangelical Lutheran Deaconess Hospital Flensburg and the Society for Energy and Climate Protection Schleswig-Holstein (Gesellschaft für Energie und Klimaschutz Schleswig-Holstein GmbH, EKSH), and the Evangelic-Lutheran Deaconesses' Hospital Flensburg, grant number 8/12-40.

Institutional Review Board Statement: Not applicable.

Informed Consent Statement: Not applicable.

Data Availability Statement: This study did not report any data.

Acknowledgments: The authors gratefully acknowledge the support of the proofreaders, in particular, for language support.

Conflicts of Interest: The authors declare no conflict of interest.

\section{Nomenclature}

\begin{tabular}{lll}
\multicolumn{2}{l}{ Roman Symbols } \\
Symbol & Unit & Description \\
$B$ & e.g., J, W & $\begin{array}{l}\text { Demand } \\
c_{p}\end{array}$ \\
$E$ & $\mathrm{~J} /(\mathrm{kgK})$ & Specific isobaric heat capacity \\
$F_{P h O}^{B}$ & $\mathrm{~J}$ & Exergy \\
$H$ & - & PhO factor, based on demand \\
$H_{i}$ & $\mathrm{~J}$ & Enthalpy \\
& $\mathrm{J} / \mathrm{kg}$ & Lower calorific value
\end{tabular}




\begin{tabular}{|c|c|c|}
\hline$H_{S}$ & $\mathrm{~J} / \mathrm{kg}$ & Gross calorific value \\
\hline$m$ & $\mathrm{~kg}$ & Mass \\
\hline$Q$ & $\mathrm{~J}$ & Heat \\
\hline$r$ & $\mathrm{~J} / \mathrm{kg}$ & Specific condensation enthalpy \\
\hline$S$ & $\mathrm{~J}$ & Entropy \\
\hline$T$ & K & Kelvin temperature \\
\hline TTD & K & Terminal temperature difference \\
\hline \multicolumn{3}{|c|}{ Greek Symbols } \\
\hline$\zeta$ & - & Exergy efficiency \\
\hline$\eta$ & - & Efficiency \\
\hline$\lambda$ & - & Air ratio \\
\hline$v_{i}$ & - & Stoichiometric number \\
\hline$\Delta \Omega_{B}$ & e.g., J, W & Avoidable losses \\
\hline \multicolumn{3}{|c|}{ Sub- and Superscript } \\
\hline Symbol & \multicolumn{2}{|c|}{ Description } \\
\hline$a m b$ & \multicolumn{2}{|c|}{ Ambient } \\
\hline ash & \multicolumn{2}{|l|}{ Ashes } \\
\hline$B$ & \multicolumn{2}{|c|}{ Fuel, demand } \\
\hline C & \multicolumn{2}{|l|}{ Carnot } \\
\hline$c g$ & \multicolumn{2}{|c|}{ Combustion gas } \\
\hline $\mathrm{CO}$ & \multicolumn{2}{|c|}{ Incompletely burned } \\
\hline cond & \multicolumn{2}{|c|}{ Condensate } \\
\hline$E x$ & \multicolumn{2}{|l|}{ Exergy } \\
\hline$f g$ & \multicolumn{2}{|c|}{ Flue gas (exhaust) } \\
\hline$f$ & \multicolumn{2}{|c|}{ Combustion } \\
\hline$H P$ & \multicolumn{2}{|c|}{ Heat pump } \\
\hline ind & \multicolumn{2}{|c|}{ Process-independent } \\
\hline$i r r$ & \multicolumn{2}{|c|}{ Irreversible } \\
\hline$l$ & \multicolumn{2}{|c|}{ Loss; for air: dry air } \\
\hline$m$ & \multicolumn{2}{|c|}{ Mean, average } \\
\hline$N$ & \multicolumn{2}{|c|}{ Useful part (e.g., of heat) } \\
\hline $\mathrm{PhO}$ & \multicolumn{2}{|c|}{ Physical optimum } \\
\hline$q$ & \multicolumn{2}{|l|}{ Heat } \\
\hline$S$ & \multicolumn{2}{|l|}{ Surface } \\
\hline SOP & \multicolumn{2}{|c|}{ Standard operating point } \\
\hline tot & \multicolumn{2}{|c|}{ Total, overall } \\
\hline$\dot{\square}$ & \multicolumn{2}{|c|}{ Differentiation by time } \\
\hline
\end{tabular}

\section{References}

1. Volta, D. Das Physikalische Optimum als Basis von Systematiken zur Steigerung der Energie- und Stoffeffizienz von Produktionsprozessen. Ph.D. Thesis, TU Clausthal, Clausthal-Zellerfeld, Germany, 5 December 2014.

2. Keichel, C. Methode der grenzwertorientierten Bewertung-Energie- und Ressourceneffizienz von Gesamtbetriebsweisen. Ph.D. Thesis, TU Clausthal, Clausthal-Zellerfeld, Germany, 24 April 2017.

3. VDI 4663, Blatt 1. Bewertung von Energie- und Stoffeffizienz-Methodische Anwendung des Physikalischen Optimums; Verein Deutscher Ingenieure (VDI): Düsseldorf, Germany, 2013.

4. Wenzel, P.; Radgen, P.; Westermeyer, J. Comparing Exergy Analysis and Physical Optimum Method Regarding an Induction Furnace. Energies 2021, 14, 1621. [CrossRef]

5. Kerpen, L.; Schmidt, A.; Sankol, B. Differentiating the Physical Optimum from the Exergetic Evaluation of a Methane Com-bustion Process. Energies 2021, 14, 3419. [CrossRef]

6. Baehr, H.D. Thermodynamik, 16th ed.; Springer: Berlin/Heidelberg, Germany, 2016.

7. Waide, P.; Brunner, C.U. Energy-Efficiency Policy Opportunities for Electric Motor-Driven Systems-Working Paper; International Energy Agency: Paris, France, 2011.

8. Cerbe, G.; Lendt, B. Grundlagen der Gastechnik, 8th ed.; Hanser Fachbuchverlag: München, Germany, 2017. 